\title{
Antifungal Effects of Silver Nanoparticles Against Various Plant Pathogenic Fungi and its Safety Evaluation on
}

\section{Drosophila melanogaster}

\author{
Pankaj K. Tyagi ${ }^{1 *}$ (D), Rashmi Mishra ${ }^{1}$, Fahad Khan ${ }^{1}$, Dhriti Gupta ${ }^{1}$, Deepak Gola ${ }^{1(\mathbb{C})}$ \\ 1 Noida Institute of Engineering and Technology, Greater Noida, Uttar Pradesh, India \\ * Correspondence authors e-mail address: pktgenetics@gmail.com;
}

Scopus Author ID: 34881106500

Received: 11.05.2020; Revised: 24.05.2020; Accepted: 24.05.2020; Published: 27.05.2020

\begin{abstract}
In the present study, silver nanoparticles (Ag-NPs) were synthesized by a chemical and biological method. Further, nanoparticles were characterized for their morphological feature using techniques like UV-Visible, TEM, XRD, and zeta potential. Sharp UV-visible absorption maximum at 410 was observed for biological synthesized silver nanoparticles (Bio-AgNPs), whereas for chemical synthesized silver nanoparticles (CH-AgNPs) peak was observed at $414 \mathrm{~nm}$. TEM micrograph confirmed the formation of spherical nanoparticles dominantly via both protocols with an average size of nanoparticles was $50 \mathrm{~nm}$ and $25 \mathrm{~nm}$ for CH-AgNPs and Bio-NPs, respectively. Further, the antimicrobial potential of AgNPs was evaluated at different concentrations (25-100 ppm), against three pathogenic plant fungus plant (Alternaria solani, Corynespora cassiicola, and Fusarium spp.), in two different fungal media in term of inhibition of radial growth. Up to $100 \%$ inhibition for Alternaria solani and Fusarium spp. and 85\% inhibition for Corynespora cassiicola was observed at $100 \mathrm{ppm}$ AgNPs concentration on potato dextrose agar (PDA). Further, exposure of AgNPs on Drosophila melanogaster confirmed that Bio-AgNPs are nontoxic as compared to $\mathrm{CH}$-AgNPS. Hence it can be concluded that Bio-AgNPs are safe to use due to their nontoxic nature.
\end{abstract}

Keywords: Silver nanoparticles; antifungal; Drosophila melanogaster

(C) 2020 by the authors. This article is an open-access article distributed under the terms and conditions of the Creative Commons Attribution (CC BY) license (https://creativecommons.org/licenses/by/4.0/).

\section{Introduction}

Nanotechnology research has wide applications in the field of medical sciences, agriculture, electronic, cosmetics, biosensor, etc. [1-6]. Multiple studies utilize the different methods for the synthesis of nanoparticles, which come under either of these three categories physical, chemical, and biological [7-11]. The methods used for the synthesis of nanoparticles significantly affect the shape and size of the metallic nanoparticles [12-14]. Biological methods utilize plants, fungi, bacteria, and algae for the formation of nanoparticles and are nontoxic [15-17]. On the other hand, the main drawback of the physical and chemical protocol for the formation of nanoparticles is the cost and involvement of toxic chemicals [18].

Silver is well known for its antimicrobial potential [19,20]. However, in the form of nanoparticles, the antimicrobial activity of silver can be much superior [18]. The synthesis and antimicrobial potential of silver nanoparticles synthesize via multiple chemicals as well as biological entities (plant leaf, fungus, bacteria, etc.) are studied widely [15,19-21]. The way in which the utility of silver nanoparticles is increasing day by day, different methods of nanoparticles are evolving in terms of less toxic chemical involvement, cost-effectiveness, and 
stability of nanoparticles. In the present study, two different methods (chemical and biological) have been employed, and comparative analysis in terms of morphology (size and shape) and antimicrobial potential against plant pathogenic fungus (Alternaria solani, Corynespora cassiicola, and Fusarium spp) was performed. Further, the toxicity of nanoparticles was examined on Drosophila melanogaster in terms of fecundity, hatchability, and viability. In addition to this, the morphology of the nanoparticles was examined via TEM (Transmission electron microscopy), XRD (X-ray powder diffraction), and zeta potential. The novelty of this work is the comparative analysis of $\mathrm{CH}-\mathrm{AgNPs}$ and Bio-AgNPs, that help us to generate information about their antimicrobial as well as toxic nature.

\section{Materials and Methods}

\subsection{Synthesis of nanoparticles.}

For the biological synthesis of nanoparticles (Bio-AgNPs), fresh leaves were plugged from the plant, washed with distilled water, and dried at room temperature. After drying, 100 gm of leaves were crushed into the motor pestle and to this $100 \mathrm{ml}$ distilled water was added, and the mixture was boiled at $80{ }^{\circ} \mathrm{C}$. After boiling, $95 \mathrm{ml}$ of plant leaf extract (supernatant) is obtained via filtration in a flask and to this $5 \mathrm{ml}$ of $0.75 \mathrm{mM} \mathrm{AgNO} 3$ aqueous solution was added. The mixture was incubated at $30{ }^{\circ} \mathrm{C}$ and $150 \mathrm{rpm}$ for $72 \mathrm{~h}$. For the chemical synthesis of silver nanoparticles (CH-AgNPs), 50ml AgNO3 (0.75mM) mixed thoroughly with $10 \mathrm{ml}$ trisodium citrate $(38 \mathrm{mM})$ dropwise at $90^{\circ} \mathrm{C}$. It kept for $24 \mathrm{~h}$ in the dark at room temperature. After getting the silver nanoparticles in solution (biological and chemical), solutions were lyophilized, and a stock solution of $1000 \mathrm{ppm}$ was prepared for both. Different working concentrations (10 ppm, $25 \mathrm{ppm}, 50 \mathrm{ppm}$, and $100 \mathrm{ppm})$ were prepared by diluting the original silver nanoparticles stock solution with distilled water. All AgNPs solutions were stored at $4{ }^{\circ} \mathrm{C}$ for further experiments.

\subsection{Morphological characterization of silver nanoparticles.}

The $\mathrm{CH}-\mathrm{AgNPs}$ and Bio-AgNPs in the form of colloidal solutions were primarily characterized by UV-visible spectrophotometry, Transmission Electron Microscopy (TEM), and zeta potential and for the same sample were prepared according to the previously defined protocol by [21].

\subsection{Pathogenic fungi and growth media.}

Three fungal species (Alternaria solani, Corynespora cassiicola, and Fusarium spp.) were obtained from the microbiology laboratory of NEIT (Noida Institute of Engineering and Technology, Gr, Noida). All three fungal pathogens are known to cause various diseases on vegetables and crop plants.

\subsection{Antifungal activity of nanoparticles.}

In vitro antifungal assay at different concentrations (10,25, 50, and $100 \mathrm{ppm})$ of AgNPs (biological and chemical) was performed on two types of fungi growth medium (PDA and CMA). $5 \mathrm{~mL}$ of AgNPs having different concentrations was poured into growth medium before plating in a petri dish and incubated at room temperature for $30 \mathrm{~min}$. In addition to this, control plates containing only growth media were also prepared in parallel. After incubation, plates 
were inoculated by agar plugs of uniform size containing fungi at the center of each petri dish, followed by incubation at $28 \pm 2{ }^{\circ} \mathrm{C}$ for 14 days. The radial growth of fungal mycelium was measured and recorded after the incubation period of fungi on different culture medium containing different concentrations of AgNPs. Radial inhibition was calculated when the growth of mycelia in the control plate reached the edge of the petri dish. The following equation was used for calculation of the inhibition rate $(\%)$.

Inhibition rate $(\%)=\mathrm{R}-\mathrm{r} / \mathrm{R}$

where " $\mathrm{R}$ " is the radial growth of fungal mycelia on the control plate sample, and " $r$ " is the radial growth of fungal mycelia on the plate containing AgNPs.

\subsection{In vivo toxicity analysis on Drosophila melanogaster.}

In vivo toxicity effect of $\mathrm{CH}-\mathrm{AgNPs}$ and Bio-AgNPs were investigated using the Drosophila melanogaster. Life-history traits, i.e., fecundity, hatchability, and viability parameters, were used to assess the toxic effect of nanoparticles at different concentrations (10, 25, 50, and $100 \mathrm{ppm}$ ). Nanoparticles were supplemented with the food medium of Drosophila melanogaster. Further, control food media was not supplemented with any type of nanoparticles

\section{Results and Discussion}

\section{1. $U V$-visible spectrum.}

The change in solution color is the primary indication of nanoparticle synthesis. Further, the synthesis of nanoparticles can be confirmed by the maximum absorption peak between 400 to $500 \mathrm{~nm}$, which corresponds to the sharp surface Plasmon resonance. As illustrated in Figure 1 (A and B), the maximum absorption peak was observed at $414 \mathrm{~nm}$ and $410 \mathrm{~nm}$ for $\mathrm{CH}-\mathrm{AgNPs}$ and Bio-AgNPs, respectively.
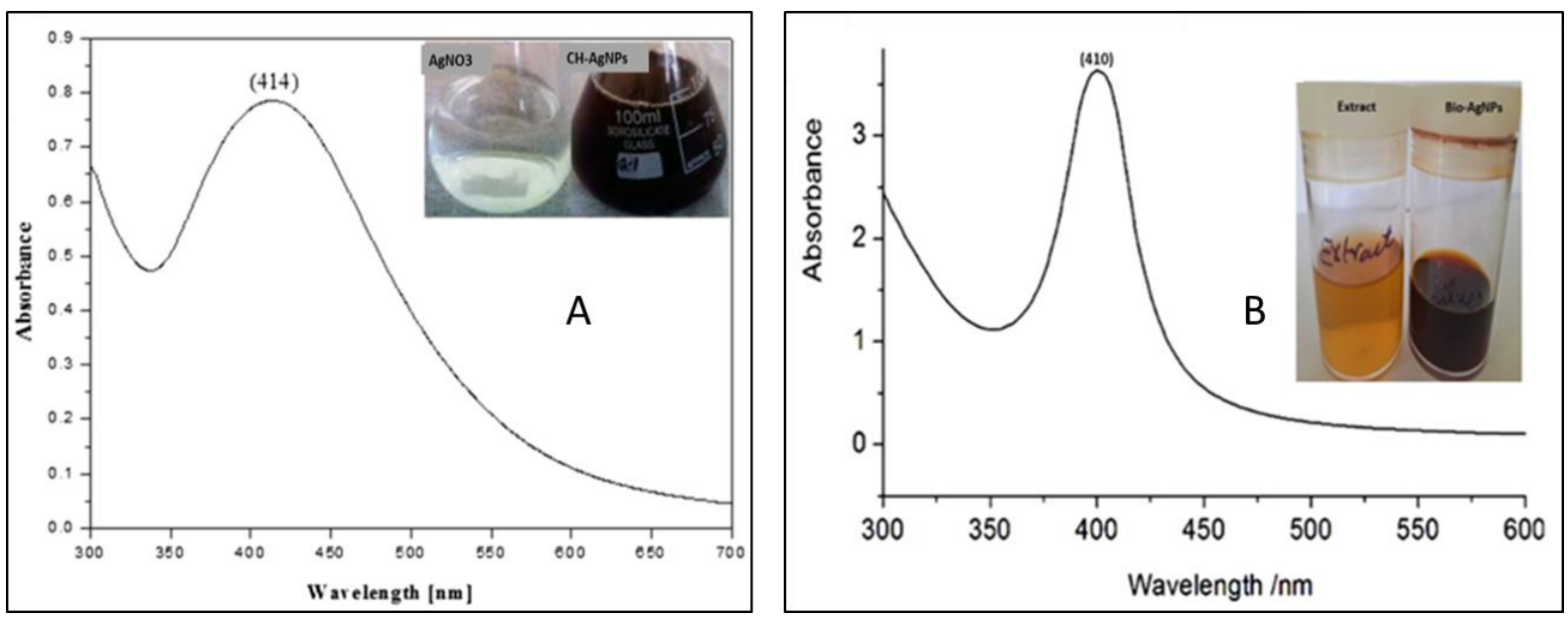

Figure 1. UV-visible spectrum of CH-AgNPs (A) and Bio-AgNPs (B).

The sharp surface Plasmon resonance peak symbolizes the formation of monodispersed nanoparticles, and the UV-visible spectrum of Bio-AgNPs showed much sharper peak as compared to the spectrum of $\mathrm{CH}-\mathrm{AgNPs}$ [18]. The possible reason behind the sharp spectrum of Bio-is the plant extract that contains a number of secondary metabolites, proteins and enzymes. All these components act as the reducing agent as well as capping during the formation of metallic nanoparticles [19]. 


\subsection{TEM.}

The TEM micrograph provides insight into the morphology feature of nanoparticles, such as shape and size. It was observed that chemically synthesized nanoparticle was cuboidal, hexagons, and spherical shapes. However, spherical shape nanoparticles were leading with an average size of $50.56 \mathrm{~nm}$ (Figure 2A). Further, no significant agglomeration was observed between the nanoparticles. On the other hand, the TEM micrograph of Bio- AgNPs indicates the synthesis of spherical and smaller nanoparticles with size ranging from 14-39 nm (Figure. 2B).
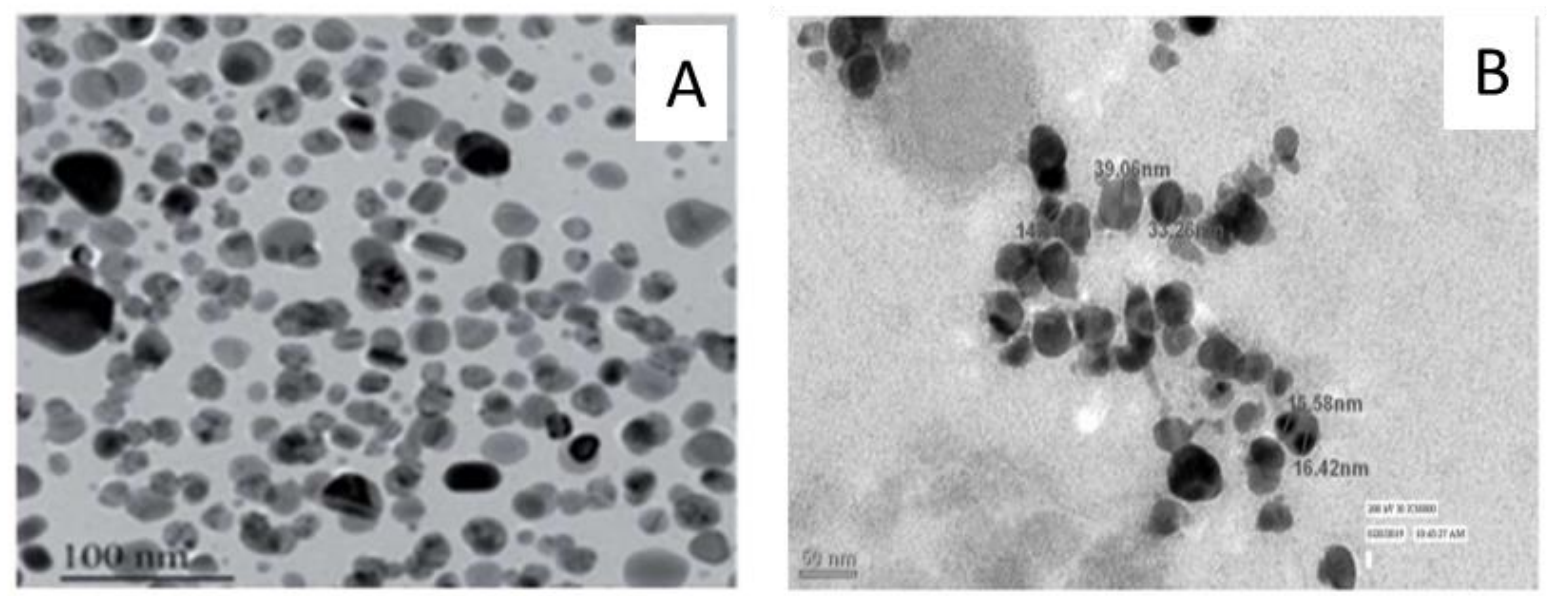

Figure 2. TEM micrograph of $\mathrm{CH}-\mathrm{AgNPs}(\mathrm{A})$ and Bio-AgNPs (B)

The morphology (size and shape) of the nanoparticles can be further enhanced by varying the concentration of chemical and plant extract in the reaction mixture, incubation time, $\mathrm{pH}$ of the reaction mixture, and the temperature of the reaction mixture [22]. The shape of the nanoparticles can directly affect its antibacterial characteristics. For example, it was investigated that triangular-shaped metallic nanoparticles were more antibacterial as compared to spherical or rod shape metallic nanoparticles [18]. This may be attributed to the sharp ends formed by the triangular shape nanoparticles as sharp ends can penetrate the cell membrane easily.

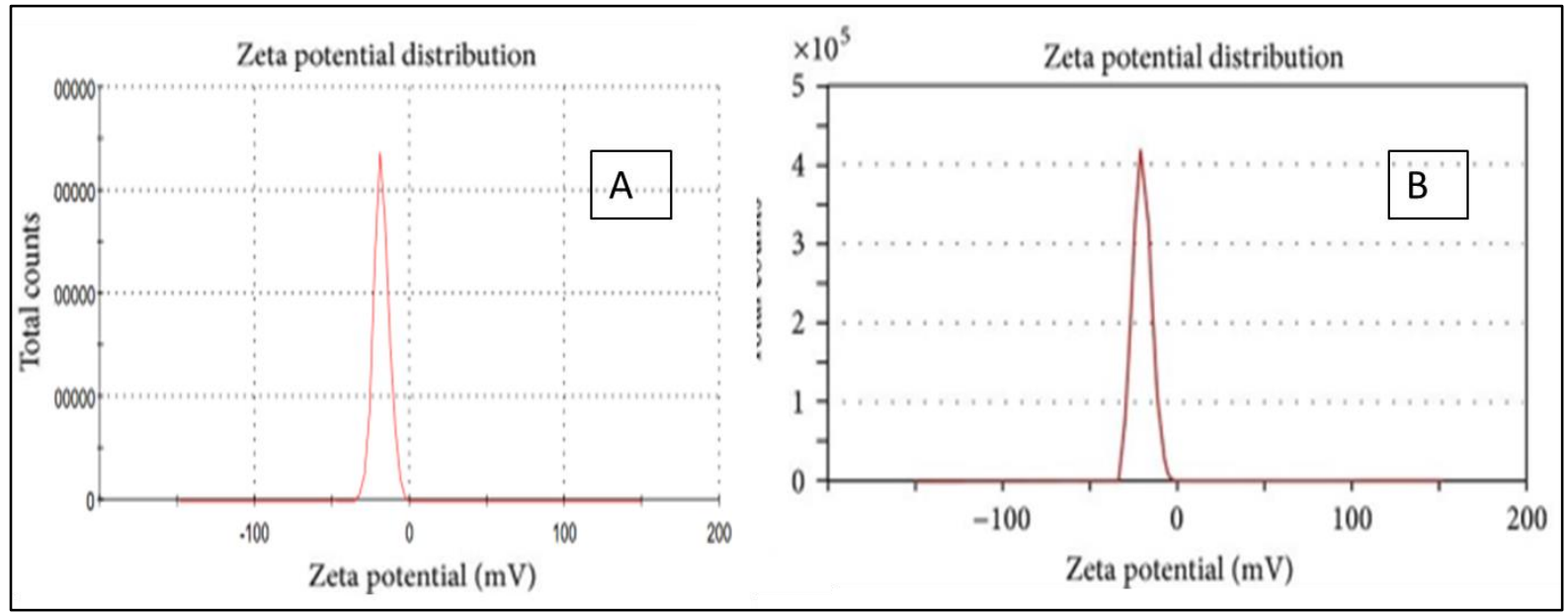

Figure 3. Zeta potential of CH-AgNPs (A) and Bio-AgNPs (B).

\subsection{Zeta potential.}

The zeta potential value for CH-AgNPs and Bio-AgNPs was $-17.24 \mathrm{mV}$ and $19.1 \mathrm{mV}$, respectively (Figure 3). It was observed that negative charge exists between the biologically as 
well as chemically synthesized AgNPs, indicating the stable synthesis. This negative charge value will cause repulsion between AgNPs and hence will reduce their aggregation tendency (Tyagi et al., 2019). A similar negative zeta potential value of $-7.66 \mathrm{mV}$ was detected for BioAgNPs produced from Pedalium murex leaf extract [23]. Table 1 represents the morphological characteristics of CH-AgNPs and Bio-AgNPs.

Table 1. Characteristics of silver nanoparticles used in this study.

\begin{tabular}{l|l|l|l|l}
$\begin{array}{l}\text { Type of } \\
\text { nanoparticles }\end{array}$ & $\begin{array}{l}\text { Physical } \\
\text { Characteristics }\end{array}$ & $\begin{array}{l}\text { Range of Particles } \\
\text { Size (nm) }\end{array}$ & $\begin{array}{l}\text { UV-vis peak range } \\
(\mathbf{n m})\end{array}$ & $\begin{array}{l}\text { Zeta potential size } \\
(\mathbf{m V})\end{array}$ \\
\hline CH-AgNPs & $\begin{array}{l}\text { Reddish brown } \\
\text { colloidal suspension }\end{array}$ & $15-60$ & 414 & -17.24 \\
\hline Bio-AgNPs & $\begin{array}{l}\text { Dark brown colloidal } \\
\text { suspension }\end{array}$ & $14-39$ & 410 & -19.10
\end{tabular}

\subsection{In vitro antifungal analysis.}

Table 2 illustrate the rate of inhibition (\%) of Alternaria solani, Corynespora cassiicola, and Fusarium spp by CH-AgNPs and Bio-AgNPs at different concentration in PDA and CMA media.

Table 2. Inhibitory rate $(\%)$ caused by $\mathrm{CH}-\mathrm{AgNPs}$ and AgNPs against three plant pathogenic fungi on PDA and

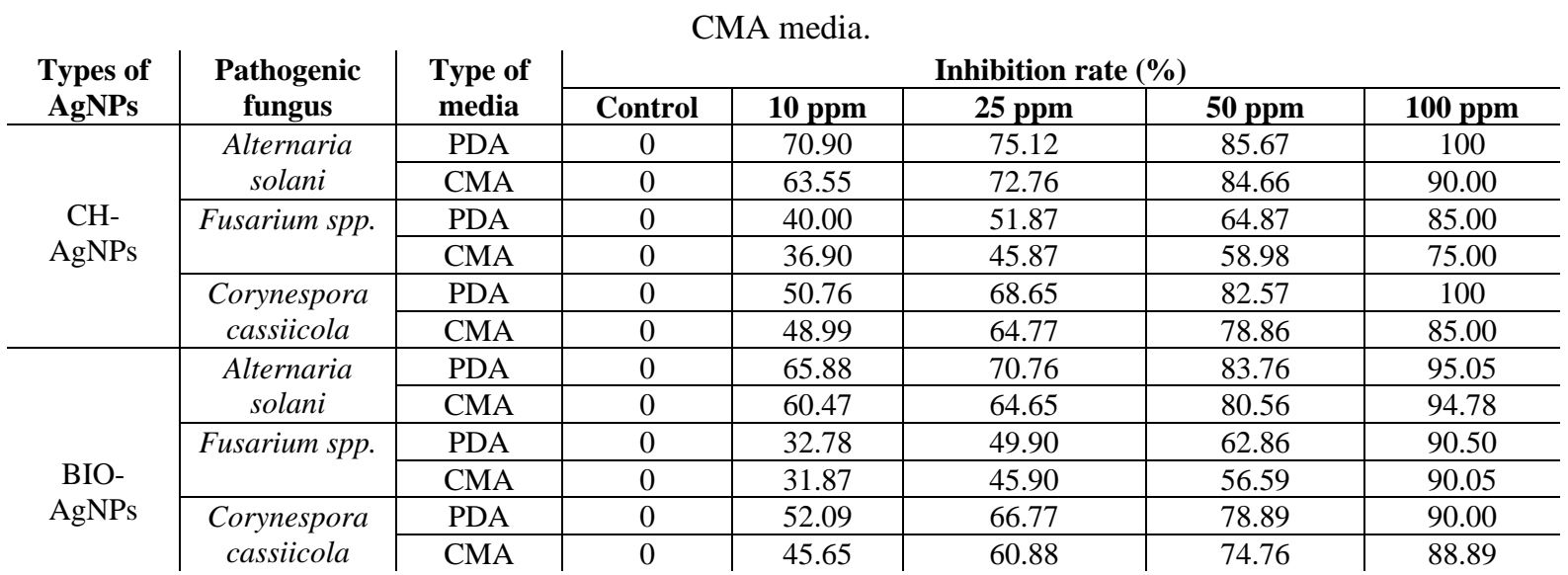

*Inhibition rates were determined based on 5 replicates of both experiment

Absolute inhibition (100\%) was observed against Alternaria solani, and Corynespora cassiicola in PDA media via CH-AgNPs, whereas Bio-AgNPs showed up to $95 \%$ and $90 \%$ inhibition at the same concentration, respectively. The lowest level of inhibition (85\%) at 100 ppm on the PDA was observed with Fusarium spp. by CH-AgNPs followed by Corynespora cassiicola (90\%) against Bio-AgNPs. On the other hand, 90\% inhibition growth was observed for Alternaria solani, followed by Fusarium spp. (85\%) and Corynespora cassiicola (75\%) at $100 \mathrm{ppm}$ concentration in CMS medium. It was observed that as the concentration of nanoparticles in the growth media increases, the growth inhibition rate percentage of fungus increase in all the cases (Figure 4).

The maximum increase in inhibition rate, i.e., is $57 \%$, and $58 \%$ was observed for Fusarium spp. against Bio-AgNPs as the concentration increased from $10 \mathrm{ppm}$ to $100 \mathrm{ppm}$ in the PDA and CMA media, respectively. On the other hand, the minimum increase in inhibition rate i.e, is $29 \%$ and $26 \%$ was observed for Alternaria solani against CH-AgNPs as the concentration increased from $10 \mathrm{ppm}$ to $100 \mathrm{ppm}$ in the PDA and CMA media, respectively. Up to $90 \%$ inhibition was observed in the growth of Fusarium solani at $20 \mu \mathrm{g} / \mathrm{ml}$ of AgNPs 
[24]. In another study, inhibition up to $71 \%-77 \%$ was observed with different strains of $R$. solanion CDA medium at $0.0019 \mathrm{~mol} / \mathrm{L}$ of AgNPs [25].

\section{CH-AgNPs}

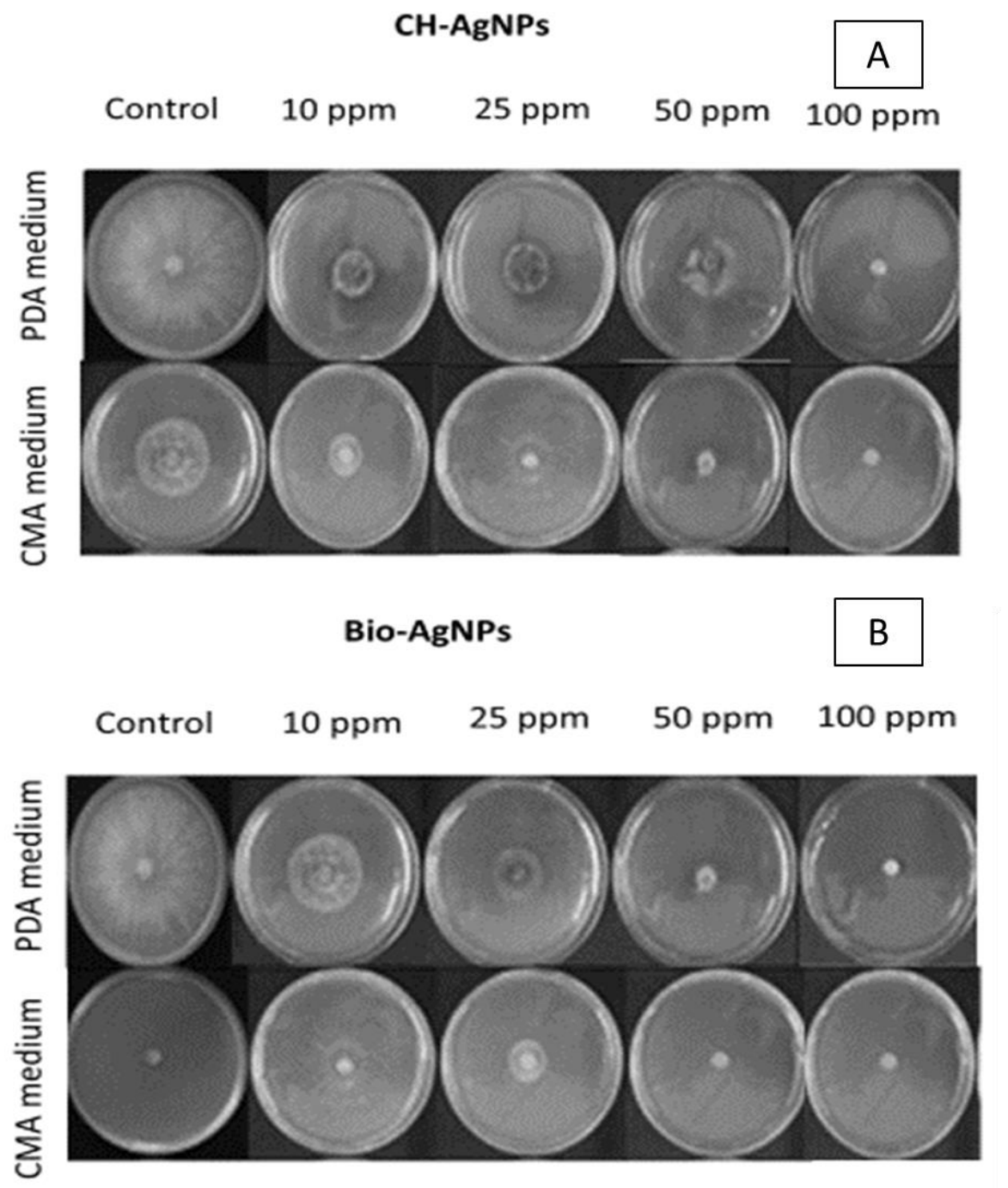

Figure 4. In vitro inhibition effects of $\mathrm{CH}-\mathrm{AgNPs}$ and Bio-AgNPs against fungus on PDA and CMA medium.

The results indicate that both $\mathrm{CH} / \mathrm{Bio}-\mathrm{AgNPs}$ are capable of inhibiting the growth of fungal pathogens; however, results vary according to the concentration dose and type of AgNPs (chemical/biological) exposed against fungal pathogens. Most fungi showed a high inhibition effect at a $100 \mathrm{ppm}$ concentration of silver nanoparticles. In addition to this, the results indicate that higher inhibition growth rate was observed on PDA media as compared to CMS medium. As the concentration of AgNPs increases inhibition rate increases, this might be due to the high density at which the solution was able to saturate and cohere to fungal hyphe as well as to deactivate plant pathogenic fungi.

The probable mechanism of inhibition may include loss in the ability to replicate DNA and inactivation of certain other cellular proteins as well as enzymes essential to the ATP production [21,26-28].

\subsection{In vivo toxicity analysis on Drosophila melanogaster.}

After identifying the rate of inhibition in the percentage of the CH-AgNPs and BioAgNPs, the next steps are to evaluate the toxicity of these nanoparticles. To examine the in vivo toxicity, we selected Drosophila melanogaster as the model organism. The in vivo toxic 
effects of nanoparticles on the developmental stages of Drosophila melanogaster were investigated at 4 different concentrations. The control fly laid on average 60 eggs/fly/day with $98 \%$ hatchability and $96 \%$ viability in all the replicates. It was observed that as compared to control Bio-AgNPs did not cause any significant difference in fecundity, hatchability, and viability (Figure 5, 6, and 7). The above results clearly indicate that Bio-AgNPs were nontoxic to Drosophila melanogaster. Whereas with the $\mathrm{CH}-\mathrm{AgNPs}$, reduction in fecundity, hatchability, and viability was observed as the concentration increases in the food media. Therefore, the results suggested that the Bio-AgNPs did not induce any toxicity on the developmental stages of Drosophila melanogaster.

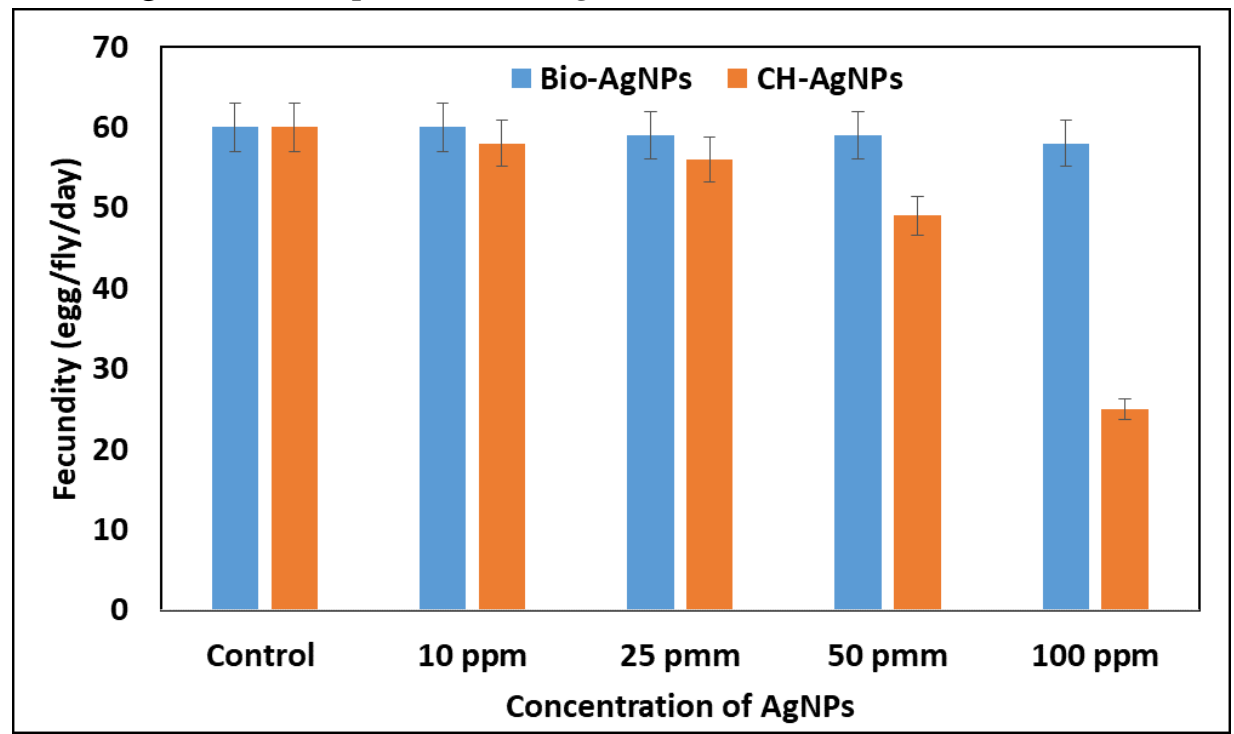

Figure 5. Fecundity of Drosophila melanogaster against Bio-AgNPs and CH-AgNPs.

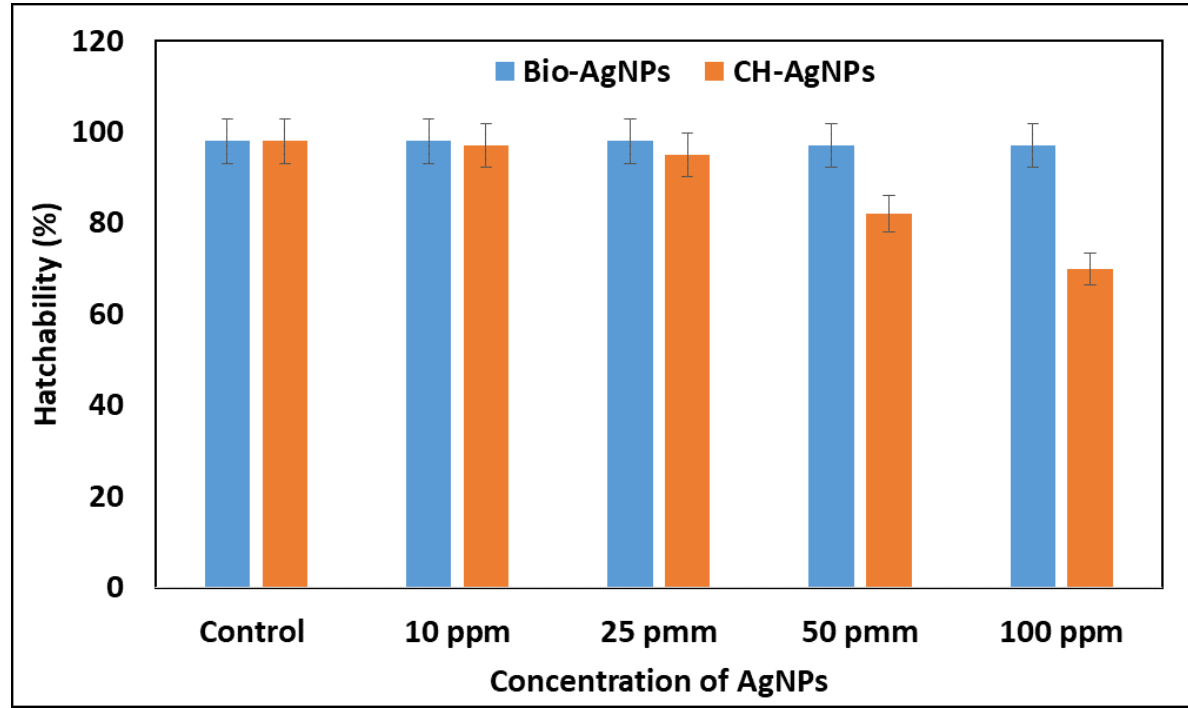

Figure 6. Hatchability of Drosophila melanogaster against Bio-AgNPs and CH-AgNPs.

On the other hand, we can say, $\mathrm{CH}-\mathrm{AgNPs}$ were toxic for Drosophila melanogaster as compare to Bio-AgNPs. It was observed that no significant change was occurred up to $25 \mathrm{ppm}$ (CH-AgNPs), but above 25 ppm fecundity, hatchability, and viability decrease significantly. Fecundity was reduced to $25 \mathrm{egg} / \mathrm{fly} /$ day from $60 \mathrm{egg} / \mathrm{fly} /$ day (control) at $100 \mathrm{ppm}$. Similarly, up to $27 \%$ and $50 \%$ reduction in hatchability and viability was observed as compared to BioAgNPs (Figures 6 and 7). The above results clearly indicate that no toxic effect was observed for Bio-AgNPs. In contrast, chemically synthesized AgNPs were toxic to Drosophila melanogaster. Hence it can be concluded that biologically synthesized nanoparticles are safe 
to use as compared to chemically synthesized nanoparticles. In summary, the Bio-AgNPs have considerable antifungal activity as well as found to be nontoxic to Drosophila melanogaster. Researchers examined the in vivo toxicity of AgNPs (purchased from Sun Innovations Corp., USA) on Drosophila and observed that ingestion of AgNPs with food medium at higher doses significantly affects the egg-laying ability as well as impaired growth of ovary [29]. Moreover, AgNPs (with Ag concentration: $20 \mathrm{mg} / \mathrm{L}$ ) were observed to cause acute toxicity in Drosophila as $50 \%$ of the tested population were incompetent to leave the pupae by hindering their development cycle [30]. De Lima et al. (2012) reviewed the comparative toxicity of chemically and biogenic generated AgNPs and concluded that biogenic AgNPS were less toxic as compared to chemically generated AgNPs [31].

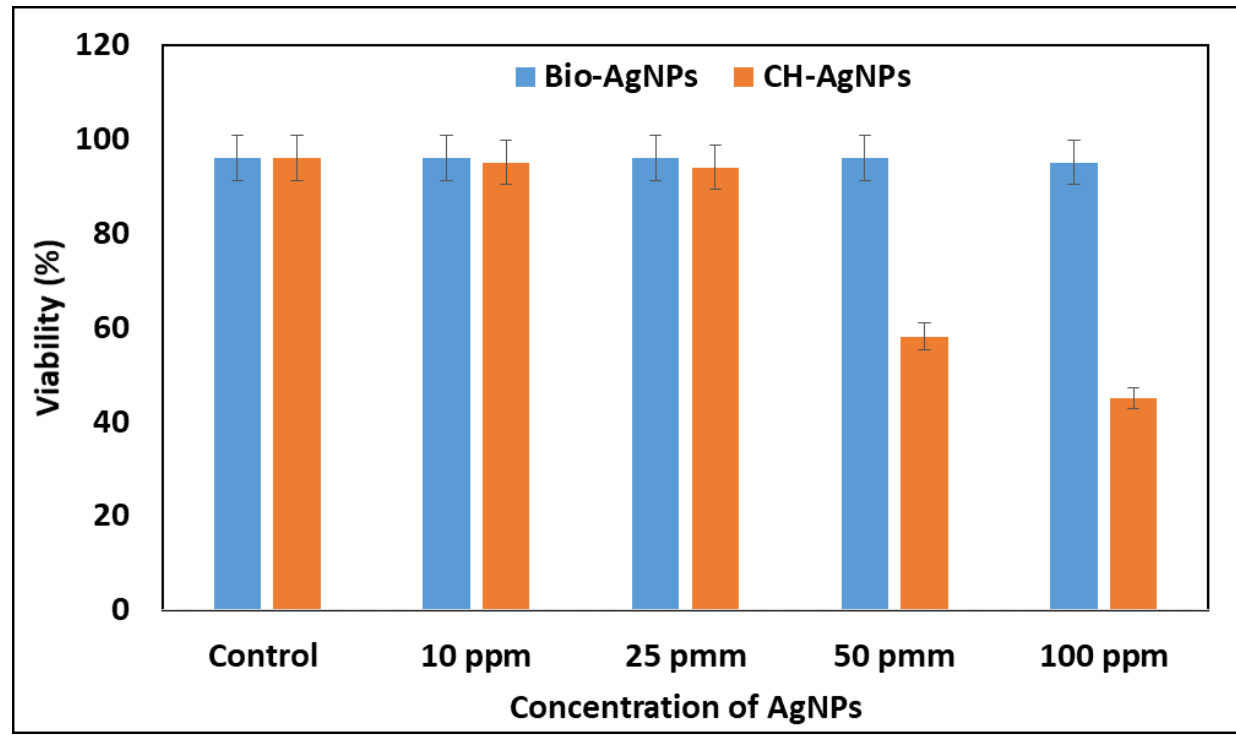

Figure 7. Viability of Drosophila melanogaster against Bio-AgNPs and CH-AgNPs.

\section{Conclusions}

The present study is a comparative analysis of AgNPs synthesizes by biological and chemical methods. It was observed that Bio-AgNPs were more stable, uniformed in shape, and smaller in size as compared to CH-AgNPs. Further, it can be concluded that in term of antifungal activity, both types of nanoparticles were showing inhibitory effect, and this inhibitory effect was dependent on AgNPs dose, type of growth media, and type of fungal species. In addition to this, Bio-AgNPs were found to be nontoxic against Drosophila melanogaster. In contrast, $\mathrm{CH}-\mathrm{AgNPs}$ were toxic, and its toxicity is dependent on the concentration of nanoparticles. So it can be concluded that Bio-AgNPs are safe to use as compared to $\mathrm{CH}-\mathrm{AgNPs}$.

\section{Funding}

This research was supported by a grant from the Collaborative Research and Innovative Programme (CRIP) funding through TEQIP-III, of Abdul Kalm Technical University (File no. AKTU/Dean-PGSR/2019/CRIP/25) Lucknow, Uttar Pradesh.

\section{Acknowledgments}

Authors acknowledged the technical support provided by Mr. Raguvendra (Lab technician, Department of Biotechnology). Authors also highly thankful to Editor of this Journal for their valuable suggestions for streghting this MS. 


\section{Conflicts of Interest}

\section{The authors declare no conflict of interest.}

\section{References}

1. Chen, X.; Guo, Z.; Tang, Y.; Shen, Y.; Miao, P. A highly sensitive gold nanoparticle-based electrochemical aptasensor for theophylline detection. Analytica Chimica Acta 2018, 999, 54-59, https://doi.org/10.1016/j.aca.2017.10.039.

2. Keijok, W.J.; Pereira, R.H.A.; Alvarez, L.A.C.; Prado, A.R.; da Silva, A.R.; Ribeiro, J.; de Oliveira, J.P.; Guimarães, M.C.C. Controlled biosynthesis of gold nanoparticles with Coffea arabica using factorial design. Scientific Reports 2019, 9, https://doi.org/10.1038/s41598-019-52496-9.

3. Rajkumar, S.; Prabaharan, M. Multi-functional core-shell Fe3O4@ Au nanoparticles for cancer diagnosis and therapy. Colloids and Surfaces B: Biointerfaces 2019, 174, 252-259, https://doi.org/10.1016/j.colsurfb.2018.11.004.

4. Ribeiro, A.P.C.; Anbu, S.; Alegria, E.C.B.A.; Fernandes, A.R.; Baptista, P.V.; Mendes, R.; Matias, A.S.; Mendes, M.; Guedes da Silva, M.F.C.; Pombeiro, A.J.L. Evaluation of cell toxicity and DNA and protein binding of green synthesized silver nanoparticles. Biomedicine \& Pharmacotherapy 2018, 101, 137-144, https://doi.org/10.1016/j.biopha.2018.02.069.

5. Gaur, P.K.; Pal, H.; Puri, D.; Kumar, N.; Shanmugam, S.K. Formulation and development of hesperidin loaded solid lipid nanoparticles for diabetes. Biointerface Research in Applied Chemistry 2020, 10, 47284733, https://doi.org/10.33263/BRIAC101.728733.

6. Bayoumy, A.M.; Elhaes, H.; Osman, O.; Kholmurodov, K.T.; Hussein, T.; Ibrahim, M.A. Effect of nano metal oxides on heme molecule: Molecular and biomolecular approaches. Biointerface Research in Applied Chemistry 2019, 10, 4837-4845, https://doi.org/10.33263/BRIAC101.837845.

7. Mousavi-Khattat, M.; Keyhanfar, M.; Razmjou, A. A comparative study of stability, antioxidant, DNA cleavage and antibacterial activities of green and chemically synthesized silver nanoparticles. Artificial Cells, Nanomedicine, and Biotechnology 2018, 46, $\quad$ S1022-S1031, https://doi.org/10.1080/21691401.2018.1527346.

8. Ullah, I.; Cosar, G.; Abamor, E.S.; Bagirova, M.; Shinwari, Z.K.; Allahverdiyev, A.M. Comparative study on the antileishmanial activities of chemically and biologically synthesized silver nanoparticles (AgNPs). 3 Biotech 2018, 8, 1-8. https://doi.org/10.1007/s13205-018-1121-6.

9. Girilal, M.; Mohammed Fayaz, A.; Elumalai, L.K.; Sathiyaseelan, A.; Gandhiappan, J.; Kalaichelvan, P.T. Comparative Stress Physiology Analysis of Biologically and Chemically Synthesized Silver Nanoparticles on Solanum Lycopersicum L. Colloid and Interface Science Communications 2018, 24, 1-6, https://doi.org/10.1016/j.colcom.2018.02.005.

10. Abdel-Aziz, H.M.M.; Rizwan, M. Chemically synthesized silver nanoparticles induced physio-chemical and chloroplast ultrastructural changes in broad bean seedlings. Chemosphere 2019, 235, 1066-1072, https://doi.org/10.1016/j.chemosphere.2019.07.035.

11. Bafana, A.; Kumar, S.V.; Temizel-Sekeryan, S.; Dahoumane, S.A.; Haselbach, L.; Jeffryes, C.S. Evaluating microwave-synthesized silver nanoparticles from silver nitrate with life cycle assessment techniques. Science of The Total Environment 2018, 636, 936-943, https://doi.org/10.1016/j.scitotenv.2018.04.345.

12. Acharya, D.; Singha, K.M.; Pandey, P.; Mohanta, B.; Rajkumari, J.; Singha, L.P. Shape dependent physical mutilation and lethal effects of silver nanoparticles on bacteria. Scientific Reports 2018, 8, https://doi.org/10.1038/s41598-017-18590-6.

13. Abramenko, N.B.; Demidova, T.B.; Abkhalimov, E.V.; Ershov, B.G.; Krysanov, E.Y.; Kustov, L.M. Ecotoxicity of different-shaped silver nanoparticles: Case of zebrafish embryos. Journal of Hazardous Materials 2018, 347, 89-94, https://doi.org/10.1016/j.jhazmat.2017.12.060.

14. Chakraborty, I.; Feliu, N.; Roy, S.; Dawson, K.; Parak, W.J. Protein-Mediated Shape Control of Silver $\begin{array}{lllll}\text { Nanoparticles. } & \text { Bioconjugate } & \text { Chemistry } & \text { 12618, } & \text { 29, }\end{array}$ https://doi.org/10.1021/acs.bioconjchem.8b00034.

15. Hariram, M.; Vivekanandhan, S.; Ganesan, V.; Muthuramkumar, S.; Rodriguez-uribe, A.; Mohanty, A.K.; Misra, M. Tecoma stans flower extract assisted biogenic synthesis of functional Ag-Talc nanostructures for antimicrobial applications. Bioresource Technology Reports 2019, https://doi.org/10.1016/J.BITEB.2019.100298.

16. Massironi, A.; Morelli, A.; Grassi, L.; Puppi, D.; Braccini, S.; Maisetta, G.; Esin, S.; Batoni, G.; Della Pina, C.; Chiellini, F. Ulvan as novel reducing and stabilizing agent from renewable algal biomass: Application to green synthesis of silver nanoparticles. Carbohydrate Polymers 2019, 203, 310-321, https://doi.org/10.1016/J.CARBPOL.2018.09.066.

17. Saratale, R.G.; Benelli, G.; Kumar, G.; Kim, D.S.; Saratale, G.D. Bio-fabrication of silver nanoparticles using the leaf extract of an ancient herbal medicine, dandelion (Taraxacum officinale), evaluation of their antioxidant, anticancer potential, and antimicrobial activity against phytopathogens. Environmental Science and Pollution Research 2018, 25, 10392-10406, https://doi.org/10.1007/s11356-017-9581-5. 
18. Tyagi, S.; Tyagi, P.K.; Gola, D.; Chauhan, N.; Bharti, R.K. Extracellular synthesis of silver nanoparticles using entomopathogenic fungus: characterization and antibacterial potential. SN Applied Sciences 2019, 1, https://doi.org/10.1007/s42452-019-1593-y.

19. Chauhan, N.; Tyagi, A.K.; Kumar, P.; Malik, A. Antibacterial Potential of Jatropha curcas Synthesized Silver Nanoparticles against Food Borne Pathogens. Frontiers in Microbiology 2016, 7, https://doi.org/10.3389/fmicb.2016.01748.

20. Buszewski, B.; Railean-Plugaru, V.; Pomastowski, P.; Rafińska, K.; Szultka-Mlynska, M.; Golinska, P.; Wypij, M.; Laskowski, D.; Dahm, H. Antimicrobial activity of biosilver nanoparticles produced by a novel Streptacidiphilus durhamensis strain. Journal of Microbiology, Immunology and Infection 2018, 51, 45-54, https://doi.org/10.1016/J.JMII.2016.03.002.

21. Pugazhendhi, A.; Prabakar, D.; Jacob, J.M.; Karuppusamy, I.; Saratale, R.G. Synthesis and characterization of silver nanoparticles using Gelidium amansii and its antimicrobial property against various pathogenic bacteria. Microbial Pathogenesis 2018, 114, 41-45, https://doi.org/10.1016/j.micpath.2017.11.013.

22. Ji, X.; Song, X.; Li, J.; Bai, Y.; Yang, W.; Peng, X. Size Control of Gold Nanocrystals in Citrate Reduction: The Third Role of Citrate. Journal of the American Chemical Society 2007, 129, 13939-13948, https://doi.org/10.1021/ja074447k.

23. Anandalakshmi, K.; Venugobal, J.; Ramasamy, V. Characterization of silver nanoparticles by green synthesis method using Pedalium murex leaf extract and their antibacterial activity. Applied Nanoscience 2016, 6, 399-408, https://doi.org/10.1007/s13204-015-0449-z.

24. Khatami, M.; Sharifi, I.; Nobre, M.A.L.; Zafarnia, N.; Aflatoonian, M.R. Waste-grass-mediated green synthesis of silver nanoparticles and evaluation of their anticancer, antifungal and antibacterial activity. Green Chemistry Letters and Reviews 2018, 11, 125-134, https://doi.org/10.1080/17518253.2018.1444797.

25. Elgorban, A.M.; El-Samawaty, A.E.-R.M.; Yassin, M.A.; Sayed, S.R.; Adil, S.F.; Elhindi, K.M.; Bakri, M.; Khan, M. Antifungal silver nanoparticles: synthesis, characterization and biological evaluation. Biotechnology \& Biotechnological Equipment $\quad$ 2016, $\quad 30, \quad 56-62$, https://doi.org/10.1080/13102818.2015.1106339.

26. Yamanaka, M.; Hara, K.; Kudo, J. Bactericidal Actions of a Silver Ion Solution on Escherichia coli, Studied by Energy-Filtering Transmission Electron Microscopy and Proteomic Analysis. Applied and Environmental Microbiology 2005, 71, 7589-7593. https://doi.org/10.1128/AEM.71.11.7589-7593.2005.

27. Durán, N.; Durán, M.; de Jesus, M.B.; Seabra, A.B.; Fávaro, W.J.; Nakazato, G. Silver nanoparticles: A new view on mechanistic aspects on antimicrobial activity. Nanomedicine: Nanotechnology, Biology and Medicine 2016, 12, 789-799, https://doi.org/10.1016/j.nano.2015.11.016.

28. Yan, X.; He, B.; Liu, L.; Qu, G.; Shi, J.; Hu, L.; Jiang, G. Antibacterial mechanism of silver nanoparticles in Pseudomonas aeruginosa: proteomics approach. Metallomics 2018, 10, 557-564, https://doi.org/10.1039/c7mt00328e.

29. Raj, A.; Shah, P.; Agrawal, N. Dose-dependent effect of silver nanoparticles (AgNPs) on fertility and survival of Drosophila: An in-vivo study. PLOS ONE 2017, 12, https://doi.org/10.1371/journal.pone.0178051.

30. Panacek, A.; Prucek, R.; Safarova, D.; Dittrich, M.; Richtrova, J.; Benickova, K.; Zboril, R.; Kvitek, L. Acute and Chronic Toxicity Effects of Silver Nanoparticles (NPs) on Drosophila melanogaster. Environmental Science \& Technology 2011, 45, 4974-4979, https://doi.org/10.1021/es104216b.

31. de Lima, R.; Seabra, A.B.; Durán, N. Silver nanoparticles: a brief review of cytotoxicity and genotoxicity of chemically and biogenically synthesized nanoparticles. Journal of Applied Toxicology 2012, 32, 867-879, https://doi.org/10.1002/jat.2780. 